\title{
The importance of patient and public involvement (PPI) in the design and delivery of maternal weight management advice.
}

\section{Abstract}

Optimum gestational weight gain (GWG) is vital for successful pregnancy outcomes. However, pregnant women often feel misinformed or that interventions will not meet their needs; hence, many services report poor attendance and/or engagement ${ }^{[1]}$. PPI aims to empower those affected by an intervention, to participate in shaping the intervention ${ }^{[2,]}$. The aim of this study was to conduct PPI to gain insight into the experiences of weight management advice received during pregnancy - to inform future interventions.

We recruited two PPI representatives (PLRs) - former patients, who assisted in all aspects of the study: design, interview questions, recruitment of PPI participants and collection/analysis of feedback. The PLRs approached pregnant/postnatal women to participate. During the PPI workshop procedure, women gave verbal consent for their views and possible verbatim quotes to be used in publications/grant applications. Feedback was collected via notetaking during four group discussions (approximately 1 hour in duration), all held in community locations - two in Liverpool $(n=10 \& 5)$, two in Derry $(n=7 \& 9)$ and an interview $(n=1$, in Derry). The research team collated the transcripts, subjected them to repeated reading and thematic analysis, informed by Braun and Clarke's step-by-step guide $^{[3]}$.

Three themes were identified: 1) Weight gain is inevitable in pregnancy; 2) Healthy eating advice is important but lacks consistency and depth; 3) Expectations regarding knowledge/support. Regarding theme 1: Most women viewed weight gain as an inevitable part of pregnancy, with variable amounts of weight gain being reported. Some women viewed pregnancy as an opportunity to 'relax' previous diets - using pregnancy as an excuse to gain weight 'without feeling guilty'. Women were only weighed at their initial appointment but had no opposition to routine weighing throughout pregnancy or for research, providing they were informed about its significance and importance:

"It would be ok to be weighed if it is for baby's health."

However, they were opposed to 'weight watching':

"We live in a society where weight is such a big issue. It can be difficult putting on weight and thinking about it too much and stressing yourself."

Despite known risks associated with excess GWG, women considered weight gain 'inevitable', so deemed weight monitoring unnecessary. NICE guidance ${ }^{[4]}$ advises against routine weighing which 'may produce unnecessary anxiety with no added benefit'.However, regular weighing may help to avert excessive GWG, if conducted sensitively and with appropriate dietary/lifestyle support, versus weighing without intervention, which may cause 'stress'.

\section{Conflict of Interest}

There is no conflict of interest 